\title{
Probing the prospective FCC-he sensitivities on the electromagnetic dipole moments of the top-quark
}

\section{A. Hernández-Ruíz, ${ }^{a, *}$ M. Köksal, ${ }^{b}$ A. A. Billur ${ }^{c}$ and A. Gutiérrez-Rodríguez. ${ }^{d}$}

${ }^{a}$ Unidad Académica de Ciencias Químicas, Universidad Autónoma de Zacatecas Apartado Postal 585, 98060 Zacatecas, México.

${ }^{b}$ Deparment of Optical Engineering, Sivas Cumhuriyet University 58140, Sivas, Turkey.

${ }^{c}$ Deparment of Physics, Sivas Cumhuriyet University 58140, Sivas, Turkey.

${ }^{d}$ Unidad Académica de Física, Universidad Autónoma de Zacatecas Apartado Postal C-580, 98060 Zacatecas, México.

E-mail: mahernan@uaz.edu.mx, mkoksal@cumhuriyet.edu.tr, abillur@cumhuriyet.edu.tr, alexgu@fisica.uaz.edu.mx

The measurement of the top-quark anomalous electromagnetic couplings is one of the most important goals of the top-quark physics program in the present and future collider experiments. This would provide direct information on the non-standard interactions of the top-quark. We study a top-quark pair production scenario at the Future Circular Hadron-Electron Collider (FCC-he) through $e^{-} p \rightarrow e^{-} \gamma p \rightarrow \bar{t} v_{e} b p$ collisions, which will provide information about sensitivities on anomalous $\hat{a}_{V}$ and $\hat{a}_{A}$ couplings at a $95 \%$ C.L., and the possibility of probing new physics.

40th International Conference on High Energy physics - ICHEP2020

July 28 - August 6, 2020

Prague, Czech Republic (virtual meeting)

${ }^{*}$ Speaker 


\section{Introduction}

Since the detection of the top-quark, there has been an enormous motivation to investigate the properties and the potential of the top-quark in great detail both in production and in decay. The experimental results of the current colliders are complemented by very precise theoretical predictions in the framework of the Standard Model of particle physics and beyond. Specifically, the anomalous coupling $t \bar{t} \gamma$, which is the subject of this work, has been studied in detail at in hadron colliders and at future high-luminosity high-energetic linear electron-positron colliders [1-3].

The Standard Model prediction for the Magnetic Moment (MM) and the Electric Dipole Moment (EDM) of the top-quark, that is $a_{t}[4]$ and $d_{t}[5-7]$ reads:

$$
a_{t}=0.02, \quad d_{t}<10^{-30}(e \mathrm{~cm}) .
$$

The $a_{t}$ can be tested at current and future colliders such as the LHC, CLIC, the Large HadronElectron Collider ( $\mathrm{LHeC}$ ) and the FCC-he. In the case of the $d_{t}$, its value is strongly suppressed, and is much too hard to be observed. However, it is very attractive for probing new physics BSM. Furthermore, it is considered as a source of $\mathrm{CP}$ violation.

\section{Single top-quark production via the process $e^{-} p \rightarrow e^{-} \gamma p \rightarrow \bar{t} v_{e} b p$}

We study the dipole moments of the top-quark in a model-independent way through the single top-quark production process $e^{-} p \rightarrow e^{-} \gamma p \rightarrow \bar{t} v_{e} b p$. Fig. 1 shows the schematic diagram for the process $e^{-} p \rightarrow e^{-} \gamma p \rightarrow \bar{t} v_{e} b p$ and the Feynman diagrams contributing to the reaction $e^{-} \gamma \rightarrow \bar{t} v_{e} b$.
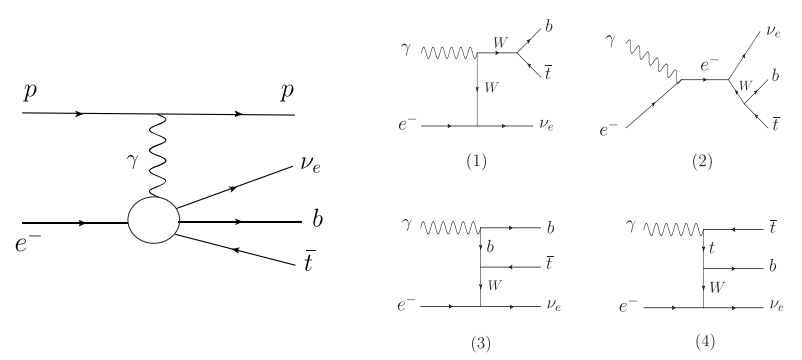

Fig. 1: A schematic diagram for the process $e^{-} p \rightarrow e^{-} \gamma p \rightarrow \bar{t} v_{e} b p$ and the Feynman diagrams the subprocess $e^{-} \gamma \rightarrow \bar{t} v_{e} b(1-4)$.

\section{Results and Conclusion}

Model-independent sensitivity estimates on the $\hat{a}_{V}$ and $\hat{a}_{A}$.

We have calculated the sensitivity of the non-standard couplings, $\hat{a}_{V}$ and $\hat{a}_{A}$, for the process $e^{-} p \rightarrow e^{-} \gamma p \rightarrow \bar{t} v_{e} b p$. We base our results on the following kinematic acceptance cuts in order to optimize the significance of the signal over all the backgrounds:

$$
\text { Cut-1: } p_{T}^{b}>20 \mathrm{GeV}, \quad \text { Cut-2: }\left|\eta^{b}\right|<2.5, \quad \text { Cut }-3: p_{T}^{v_{e}}>15 \mathrm{GeV}
$$


In Eq.(1), $p_{T}^{b}$ is the transverse momentum of the final state bottom-quark, $\eta^{b}$ is the pseudo-rapidity and $p_{T}^{v_{e}}$ is the transverse momentum of the electron-neutrino. We consider the center-of-mass energies $\sqrt{s}=7.07 \mathrm{TeV}, \sqrt{s}=10 \mathrm{TeV}$ and luminosities $\mathcal{L}=50,100,300,500,1000 \mathrm{fb}^{-1}$ with unpolarized and polarized electron beam. Furthermore, We have considered the kinematic acceptance cuts given by Eq. (1), take into account the systematic uncertainties $\delta_{\text {sys }}=0 \%, 3 \%, 5 \%$ and we follow three different confidence level (C.L.) $68 \%, 90 \%$ and $95 \%$ and to make our study more effective we perform a $\chi^{2}$ test define in Eq. (2),

$$
\chi^{2}\left(\hat{a}_{V}, \hat{a}_{A}\right)=\left(\frac{\sigma_{S M}-\sigma_{B S M}\left(\sqrt{s}, \hat{a}_{V}, \hat{a}_{A}\right)}{\sigma_{S M} \sqrt{\left(\delta_{s t}\right)^{2}+\left(\delta_{s y s}\right)^{2}}}\right)^{2} .
$$

Here $\sigma_{S M}$ is the cross-section from the SM, while $\sigma_{B S M}\left(\sqrt{s}, \hat{a}_{V}, \hat{a}_{A}\right)$ is the total cross-section which contains contributions from the SM, as well as non-standard contributions which come from the anomalous couplings $\hat{a}_{V}$ and $\hat{a}_{A} . \quad \delta_{s t}=\frac{1}{\sqrt{N_{S M}}}$ and $\delta_{s y s}$ are the statistical and systematic uncertainties.
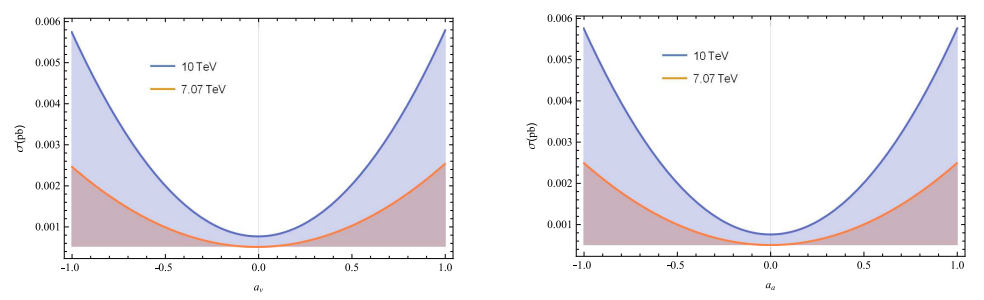

Fig. 2: The total cross sections of the process $e^{-} p \rightarrow e^{-} \gamma p \rightarrow \bar{t}_{e} b p$ as a function of $\hat{a}_{V}$ and $\hat{a}_{A}$ for center-of-mass energies of $\sqrt{s}=7.07 \mathrm{TeV}$ and $\sqrt{s}=10 \mathrm{TeV}$ at the FCC-he.

In conclusion, our sensitivity study is cut-based and uses polarized beam. We find that the total cross-section $\sigma\left(e^{-} p \rightarrow e^{-} \gamma p \rightarrow \bar{t} v_{e} b p\right)$ has a strong dependence on the anomalous couplings $\hat{a}_{V}$ and $\hat{a}_{A}$, as well as with on center-of-mass energies of the FCC-he and therefore strong sensitivity estimated are obtained on $\sigma\left(e^{-} p \rightarrow e^{-} \gamma p \rightarrow \bar{t} v_{e} b p\right)$ and $\hat{a}_{V}\left(\hat{a}_{A}\right)$. Our results show that with the process $e^{-} p \rightarrow e^{-} \gamma p \rightarrow \bar{t} v_{e} b p$ the FCC-he, the sensitivity estimated on the MM and the EDM of the top-quark can be significantly strengthened.

\section{References}

[1] A. A. Billur, M. Köksal, A. Gutiérrez-Rodríguez, M. A. Hernández-Ruíz, Eur. Phys. J. Plus, (2019) 134: 547.

[2] M. Köksal, A. A. Billur and A. Gutiérrez-Rodríguez, Adv. High Energy Phys., 6738409 (2017).

[3] A. A. Billur, M. Köksal and A. Gutiérrez-Rodríguez, Phys. Rev. D96, 056007 (2017).

[4] W. Bernreuther, et al., Phys. Rev. Lett. 95, 261802 (2005).

[5] F. Hoogeveen, Nucl. Phys. B341, 322 (1990).

[6] M. E. Pospelov and I. B. Khriplovich, Sov. J. Nucl. Phys. 53, 638 (1991).

[7] A. Soni and R. M. Xu, Phys. Rev. Lett. 69, 33 (1992). 